\title{
Centred approach to the period of anharmonic oscillators
}

\author{
Jonathan Landy ${ }^{1}$ and Re'em Sari ${ }^{2}$ \\ ${ }^{1}$ Physics and Astronomy Building, University of California Los Angeles, Los Angeles, \\ CA 90095-1547, USA \\ ${ }^{2}$ California Institute of Technology, Mail Code 130-33, Pasadena, CA 91125, USA \\ E-mail: landy@physics.ucla.edu
}

Received 20 June 2007, in final form 2 August 2007

Published 7 September 2007

Online at stacks.iop.org/EJP/28/1051

\begin{abstract}
We present a simple method for obtaining a concise series expression for the period of one-dimensional classical oscillators. The series converges well for typical potentials and is of a form that is often suitable for obtaining approximate expressions for the period valid to any order in the amplitude desired. The method is most easily applied to even potentials. However, by employing the lower turning point expansion discussed in the appendices it may readily be applied even to those potentials where the lower turning point may not be solved for explicitly in terms of the upper turning point. We demonstrate the method by obtaining expressions for the periods of both the simple pendulum and tadpole orbits.
\end{abstract}

\section{Introduction}

When a classical particle of mass $m$ is constrained to move within a one-dimensional potential well $V(x)$, the particle will undergo an oscillatory motion with characteristic period $T$ about the potential's local minimum. To obtain a lowest-order approximation for the period one may often expand the potential in a Taylor series about the minimum and drop all but the leading $\left(x-x_{0}\right)^{2}$ term, obtaining $V(x) \approx c+k\left(x-x_{0}\right)^{2} / 2$. This is the familiar harmonic potential whose period is given by the formula $T=2 \pi \sqrt{m / k}$. It should be noted, however, that not all potentials may be treated in this fashion [8].

To obtain more accurate approximations valid for large amplitudes, many other techniques have been employed. Often these techniques involve an expansion of the integrand in the following rearrangement of the conservation of energy equation [7]:

$$
T=\sqrt{2 m} \int_{x_{-}}^{x_{+}} \frac{\mathrm{d} x}{\sqrt{E-V(x)}} .
$$


Here $E$ is the energy and $x_{+}$and $x_{-}$, the turning points of the motion, are solutions to the equation $E-V(x)=0$. Hence the integrand in (1) is singular at both end points of the domain of integration.

For the restricted case where the potential is such that the integral (1) is elliptic, it is well known that a particularly simple expansion solution may be used [7]. In this paper we demonstrate that a centred expansion technique, similar to that used for the elliptic integrals, may in fact be applied to any potential which is Taylor expandable. Thus the method presented here appears to be at least as general as any previously published method for obtaining the period of anharmonic oscillators.

In contrast to the case of small amplitude oscillations, often little or no time is currently spent on the study of large amplitude motion in undergraduate courses. This is unfortunate given that an introduction to these topics would benefit students, providing preparation for possible future encounters with large amplitude oscillators in their research. Further, through the study of large amplitude motion many interesting properties of oscillators can be observed which might not otherwise be apparent through the study of small amplitude motion alone. The divergence of the period at small amplitudes for motion under potentials without a quadratic component at their local minimum and the determination of the degree to which the period of an oscillator can be specified when the potential is approximated by a polynomial are two such examples covered in this paper. The approach taken here is quite straightforward and should provide convincing evidence that a presentation on topics related to large amplitude motion could be carried out at a level appropriate for undergraduates.

\section{Expansion solution}

To solve the integral (1) we begin by factoring out the two turning point roots from the denominator of the integrand:

$$
\frac{T}{\sqrt{2 m}}=\int_{x_{-}}^{x_{+}} \frac{\mathrm{d} x}{\sqrt{\left(x_{+}-x\right)\left(x-x_{-}\right)}} R(x),
$$

where

$$
R(x) \equiv \sqrt{\frac{\left(x_{+}-x\right)\left(x-x_{-}\right)}{E-V(x)}} .
$$

Centring the integral by the change of variables $u=x-\frac{x_{-}+x_{+}}{2}$, we obtain

$$
\frac{T}{\sqrt{2 m}}=\int_{-A}^{A} \frac{\mathrm{d} u}{\sqrt{(A-u)(A+u)}} R\left(u+\frac{x_{-}+x_{+}}{2}\right),
$$

where

$$
A \equiv \frac{x_{+}-x_{-}}{2}
$$

is the amplitude of the motion. Expanding $R\left(u+\frac{x_{-}+x_{+}}{2}\right)$ in a series about $u=0$, the integral takes the following form:

$$
\frac{T}{\sqrt{2 m}}=\int_{-A}^{A} \frac{\left(a_{0}+a_{1} u+a_{2} u^{2}+\cdots\right) \mathrm{d} u}{\sqrt{(A-u)(A+u)}} .
$$

Here the coefficients $a_{i}$ of the expansion of $R\left(u+\frac{x_{-}+x_{+}}{2}\right)$ will, in general, be functions of the amplitude $A \equiv A\left(x_{+}\right)$. We can now integrate (6) term by term. Considering

$$
I_{n}=\int_{-A}^{A} \frac{u^{n} \mathrm{~d} u}{\sqrt{(A-u)(A+u)}}, \quad n \in \mathbb{N},
$$


the substitution $u=A \sin \theta$ gives

$$
I_{n}=A^{n} \int_{-\pi / 2}^{\pi / 2} \sin ^{n} \theta \mathrm{d} \theta .
$$

For $n$ odd, $I_{n}=0$. For $n \equiv 2 k$, even

$$
\begin{aligned}
I_{2 k} & =2 A^{2 k} \int_{0}^{\pi / 2} \sin ^{2 k} \theta \mathrm{d} \theta \\
& =A^{2 k} \frac{\Gamma(k+1 / 2) \Gamma(1 / 2)}{\Gamma(k+1)} .
\end{aligned}
$$

This last equality follows as the integral (9) may be reparameterized in terms of the beta function [11]. Plugging back into (6) we obtain a series expression for the period:

$$
T=\sqrt{2 m} \sum_{k=0}^{\infty} a_{2 k} A^{2 k} \frac{\Gamma(k+1 / 2) \Gamma(1 / 2)}{\Gamma(k+1)} .
$$

Equation (11) is our main result and relates the period of the motion to the derivatives of the function $R(x)$ about the centre of the motion. At this point the period is given in terms of both $x_{-}$and $x_{+}$, which, of course, are not independent variables. Thus to make practical use of this result, one must first solve for the lower turning point in terms of the upper turning point. For even potentials this task is trivial as $x_{-}=-x_{+}$, but for non-even potentials this may not always be possible. The authors are aware of only one previously demonstrated integration method which may be applied in situations such as these [10]. That technique is quite elegant but also somewhat computationally demanding. In appendix A we present an alternative approach in which we simply use expansion approximations to the lower turning point to obtain approximations to the period. The use of this turning point approximation method is particularly natural when used in conjunction with the period expansion described here.

A sufficient condition for the convergence of the series (11) is the convergence of the Taylor series expansion of $R\left(u+\frac{x_{-}+x_{+}}{2}\right)$ within the domain of integration. For if the Taylor series of $R\left(u+\frac{x_{-}+x_{+}}{2}\right)$ converges, this implies that the series

$$
\begin{aligned}
\sum_{k=0}^{\infty} a_{2 k} A^{2 k} & =\frac{1}{2} \sum_{k=0}^{\infty} a_{k}\left((-A)^{k}+A^{k}\right) \\
& =\frac{1}{2} \sum_{k=0}^{\infty} a_{k}(-A)^{k}+\frac{1}{2} \sum_{i=0}^{\infty} a_{k} A^{k}
\end{aligned}
$$

converges to $\frac{1}{2}\left(R\left(x_{-}\right)+R\left(x_{+}\right)\right)$, by the additive property of the convergent infinite series. Further, the monotonically decreasing, real sequence $\left\{\Gamma_{k}\right\}$, defined by

$$
\Gamma_{k} \equiv \frac{\Gamma(k+1 / 2)}{\Gamma(k+1)}=\frac{k-1 / 2}{k} \Gamma_{k-1},
$$

is bounded below by zero and thus converges. Therefore, both conditions of Abel's convergence test [2] are satisfied and the series solution (11) is guaranteed to converge.

The rate of convergence is clearly dependent upon the rate of convergence of the Taylor series for the function $R\left(u+\frac{x_{-}+x_{+}}{2}\right)$. Therefore, we expect that for smooth $R(u)$ our series for the period should converge rapidly. 


\section{Truncated series approximations}

By truncating the series (11) one may obtain analytic approximations to the period. In this section we demonstrate that one may state analytically the order to which these approximations are valid in the amplitude.

To determine the amplitude dependence of the terms in (11) we must look at the functional form of $R(x)$. Assuming, for now, that the potential is even, we have

$$
R(x)=\sqrt{\frac{x_{+}^{2}-x^{2}}{V\left(x_{+}\right)-V(x)}} \equiv \sqrt{\frac{1}{P\left(x, x_{+}\right)}} .
$$

We now observe the behaviour of $R(x)$ and its derivatives at $x=0$, in the limit $x_{+} \rightarrow 0$. In particular, note that when $x_{+}=0$,

$$
P(x, 0)=\frac{V(x)-V(0)}{x^{2}} .
$$

Since $V(x)$ is even and Taylor expandable this implies that the derivatives of $P\left(x, x_{+}\right)$with respect to $x$ remain finite as $x_{+} \rightarrow 0$. Now when $x=0$,

$$
P\left(0, x_{+}\right)=\frac{V\left(x_{+}\right)-V(0)}{x_{+}^{2}} .
$$

Therefore, when the potential is such that $V^{\prime \prime}(0)$ is nonzero (i.e. the potential is asymptotically harmonic), $P\left(0, x_{+}\right)$will go to some nonzero constant as $x_{+} \rightarrow 0$. As the derivatives of $R(x)$ at $x=0$ consist of sums of products of terms of the form $P\left(0, x_{+}\right)^{-j}$ and $P^{(n)}\left(0, x_{+}\right)$, it follows that the $a_{i}$ will also approach constants as $x_{+} \rightarrow 0$. This implies that the $k=m$ term in (11) behaves like $A^{2 m}$ as $x_{+} \rightarrow 0$. In this case then, an $n+1$ term, truncated approximation to the period will always be valid to order $2 n+1$ in $A$. By making use of the lower root expansion described in appendix A, a similar argument shows that the same conclusion holds for non-even, asymptotically harmonic potentials as well.

For asymptotically anharmonic potentials, the order of the amplitude dependence of the terms in (11) will not increase as the index increases. For example, for potentials of the form $V(x)=x^{2 m}$, each term in the series (11) is proportional to the same power of $x_{+}$, this being $\frac{1}{x_{+}^{m-1}}$. It follows that for potentials of this type, the truncated series will only be valid to $O\left(x_{+}^{-m}\right)$ regardless of the number of terms retained.

For asymptotically harmonic potentials one may also obtain power series approximations for the period for which the coefficients are $A$ independent. This is done by further expanding each of the coefficients $a_{2 k} \equiv a_{2 k}(A)$ to the appropriate order in $A$. These power series are useful as they allow one to observe the essential function form of the period at appropriately small amplitudes. Explicitly, we have

$$
T=\sqrt{2 m} \sum_{l=0}^{2 n+1} \sum_{k=0}^{2\lfloor l / 2\rfloor} c_{k, l} A^{l}+O\left(A^{2 n+2}\right),
$$

with

$$
c_{k, l}=\frac{1}{(l-2 k) !} \frac{d^{l-2 k} a_{2 k}}{d A^{l-2 k}} \frac{\Gamma(k+1 / 2) \Gamma(1 / 2)}{\Gamma(k+1)} .
$$

This can be somewhat simplified for the case of even potentials, for which the coefficients $a_{2 k} \equiv a_{2 k}\left(A^{2}\right)$ depend only upon the amplitude squared. In this case only even $l$ need be considered in the sum (18).

We also remark that often for non-even potentials, one of the turning points of the motion may provide a more natural parameter for expansion than does the amplitude. A power series 
valid to order $2 n+1$ in $x_{+}$, for example, may still be obtained by considering only the first $n+1$ terms in (11), provided the origin's position is such that $A=O\left(x_{+}^{1}\right)$.

In the following two sections we will briefly demonstrate these approximation methods by applying them to particular potentials.

\section{The simple pendulum}

The energy of a simple pendulum of length $L$ and mass $m$ is given by

$$
\begin{aligned}
E & =m g L(1-\cos \theta)+\frac{m L^{2}}{2} \dot{\theta}^{2} \\
& =m g L\left(1-\cos \theta_{M}\right),
\end{aligned}
$$

where $\theta$ is the angle the pendulum makes with the vertical axis, $\theta_{M}$ is the upper turning point angle and $g$ is the acceleration due to gravity. Equation (20) may be used to obtain an integral for the period with the same form as (1). We find

$$
T=\sqrt{2 m L^{2}} \int_{-\theta_{M}}^{\theta_{M}} \frac{\mathrm{d} \theta}{\sqrt{E-m g L(1-\cos \theta)}} .
$$

Carrying through the steps outlined in (6)-(11) and retaining only the first two terms, we obtain

$$
T=\sqrt{\frac{2 L}{g}} \sum_{k=0}^{1} a_{2 k} \theta_{M}^{2 k} \frac{\Gamma(k+1 / 2) \Gamma(1 / 2)}{\Gamma(k+1)}+O\left(\theta_{M}^{4}\right)
$$

with

$$
a_{0}=\frac{\theta_{M}}{\left(1-\cos \theta_{M}\right)^{\frac{1}{2}}}
$$

and

$$
a_{2}=\frac{\left(\theta_{M}^{2}+2 \cos \theta_{M}-2\right)}{4 \theta_{M}\left(1-\cos \theta_{M}\right)^{\frac{3}{2}}} .
$$

By expanding each of the $a_{i}$ to the appropriate order we then obtain the following power series expression for the period, valid to order $\theta_{M}^{3}$ :

$$
T=2 \pi \sqrt{\frac{L}{g}}\left(1+\frac{1}{16} \theta_{M}^{2}\right)+O\left(\theta_{M}^{4}\right) .
$$

This formula is consistent with previous perturbation approach derivations [4]. A comparison plot of the approximations (23) and (26) along with the numerical result for the integral (22) is shown in figure 1. After only two terms both series seem to be converging quite reasonably, especially so at smaller amplitudes, as expected. We find that the approximations (23) and (26) maintain 5\% accuracy for all amplitudes less than 2.49 and 1.91 radians, respectively. As a benchmark, we also note that the constant, harmonic approximation to the period maintains this level of accuracy for all amplitudes less than 0.89 radians.

\section{Tadpole orbits}

Our second example application comes from celestial mechanics and deals with the circular, restricted three-body problem once considered by Lagrange. In this problem a large mass $m_{1}$ 


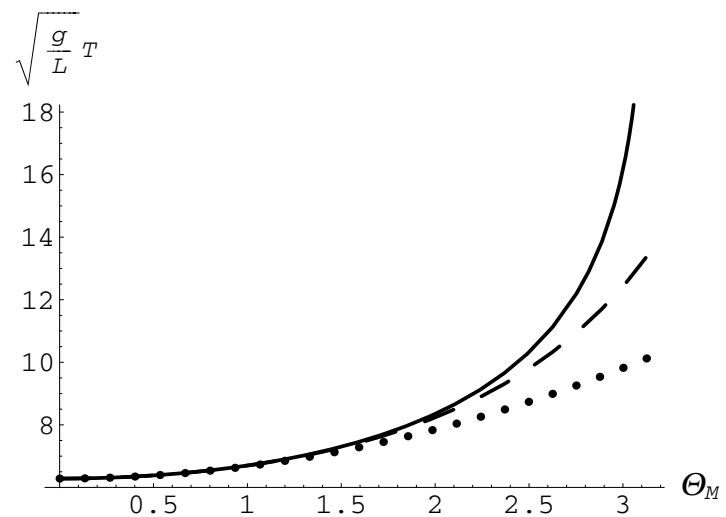

Figure 1. The two second-order approximations for the period of the pendulum, (23) (dashed line) and (26) (dotted line), are compared here to the numerical solution (solid line) for amplitudes between 0 and $\pi$.

and a secondary mass $m_{2}$ both move in circular, coplanar orbits about their common centre of mass. A third body of negligible mass is introduced to the system and orbits under the gravitational influence of the first two. Here we choose units such that $G\left(m_{1}+m_{2}\right)=1$, the constant distance between the two massive bodies is also 1, and the period of motion of the two massive bodies is $2 \pi$.

We next define a set of axes in the rotating frame such that the centre of mass is located at the origin and the secondary mass lies along the positive $x$-axis. In this frame there are five equilibrium points at which the third body may be placed and will remain fixed with respect to the coordinate axes. These are the well-known Lagrange points. Of these five points, two will be stable provided that the following condition holds [9]:

$$
\mu \equiv \frac{m_{2}}{m_{1}+m_{2}}<\frac{27-\sqrt{621}}{54} \approx 0.0385 .
$$

The two stable equilibrium points are located at $\left(\frac{1}{2}-\mu, \pm \frac{\sqrt{3}}{2}\right)$. Interestingly, each of these points, when taken with the positions of the two massive bodies, forms an equilateral triangle. Because the stability condition (27) holds for each Sun-planet pair in our solar system, as well as for many other pairs of objects such as the Earth-moon system, these equilibrium points play an important role in the dynamics of our solar system. For this reason, study of the Lagrange points remains a vigorous topic of research to this day.

When perturbed away from one of the two stable equilibrium points, the third body will undergo an orbit about the equilibrium point. The theory of these orbits is quite beautiful and has been worked out in detail $[5,9]$. Stable orbits exist which are quite elongated in the angular direction but which are also quite skinny in the radial direction. They may become so angularly elongated, in fact, that the orbit crosses the negative $x$-axis. When this occurs the orbit continues on and circles around the second stable Lagrange point. That is, the orbit now is about both stable equilibrium points and has a radius always close to 1 . Because of their shape these orbits are called horseshoe orbits. Orbits about a single Lagrange point are called tadpole orbits.

We shall use the approximation methods discussed above to get an analytic expression for the period of these orbits which should be useful at small amplitudes. To do so we take as given the following two equations valid to order $\mu$ for tadpole orbits [9]: 


$$
\begin{aligned}
& \dot{\theta}=-\frac{3}{2} \delta r \\
& \delta r^{2}+\frac{4}{3} \mu f(\theta)=\alpha .
\end{aligned}
$$

Here $\alpha$ is some constant of the motion, $\theta$ is the angle of the $x$-axis, $r \equiv 1+\delta r$, and

$$
f(\theta) \equiv \csc \frac{\theta}{2}+4 \sin ^{2} \frac{\theta}{2} .
$$

At the angular extremum, $\theta_{+}, \dot{\theta}=0$. Therefore, upon combining (28) and (29), we obtain

$$
\frac{1}{3} \dot{\theta}^{2}+\mu f(\theta)=\mu f\left(\theta_{+}\right) .
$$

Rearrangement gives

$$
T=\frac{2}{\sqrt{3 \mu}} \int_{\theta_{-}}^{\theta_{+}} \frac{\mathrm{d} \theta}{\sqrt{f\left(\theta_{+}\right)-f(\theta)}} .
$$

Solving for the sine of $\frac{\theta_{-}}{2}$ presents no particular difficulty here. Therefore, the integration of (32) may be carried out in the usual manner after the change of variables $u \equiv \sin \frac{\theta}{2}$ is applied. We present the resulting power series expression for the period below:

$$
T=\frac{2 \pi}{\sqrt{\frac{27 \mu}{4}}}\left(1+\frac{3}{16} \Delta \theta^{2}+\frac{\sqrt{27}}{32} \Delta \theta^{3}\right)+O\left(\Delta \theta^{4}\right),
$$

with

$$
\Delta \theta \equiv \theta_{+}-\frac{\pi}{3}
$$

The first term of (33) matches the standard result one obtains by linearizing the equations of motion about the equilibrium point [9]. See [6] for an alternative expansion.

This example demonstrates the usefulness of the integral approach to determining the period of oscillators. The most common alternative approach to determining the period of an oscillator is to first solve the equations of motion using a perturbative technique. The perturbative solution can then be used to approximate the period [4]. Though a perturbative solution for the orbits about the Lagrange points has been worked out [5], the calculations are quite intensive. When only the period of the motion is of interest, it is much more practical to apply the direct integration method described here.

\section{Discussion}

By factoring out the singular portion of the denominator of (1) and expanding the remainder of the integrand about the centre of the motion, we were able to obtain a series expression, with analytically calculable coefficients, for the period of one-dimensional classical oscillators. The result thus obtained is of interest in that it shows that the period is in some sense a filtered version of the Taylor series for the function $R\left(x_{-}\right)+R\left(x_{+}\right)$. The result is also of practical use as it reduces the problem of determining the period to that of the calculation of the derivatives of the function $R(x)$.

The authors are aware of two other solution methods that begin by factoring out the singular portion of the integrand as we did. Both have their merits and also their drawbacks. The method presented by Chen [3] is quite straightforward and allows for a series solution similar to ours. The amplitude dependence of the terms of his series, however, does not increase in order as the index increases. Therefore, while his series may provide accurate approximations to the period, it does not provide any immediate information on the functional 
form of the period as does ours. His method also requires two integrations for non-even potentials whereas ours requires only one. On the other hand, the method presented by Amore and Fernandez [1] allows for a series solution that converges quite well for most amplitudes under typical potentials. The coefficients of their series, however, take the form of integrals which cannot, in general, be evaluated analytically.

Among these and the many other solution techniques which have been discussed previously, the one we have presented here has allowed for a nice compromise of positive attributes: it converges relatively well for typical potentials, the coefficients of the series demonstrate a useful amplitude dependence and may be analytically calculated for any differentiable potential, it may be used in conjunction with a turning point approximation method to obtain period approximations when one cannot solve explicitly for one turning point in terms of the other, and perhaps most importantly, the technique is very straightforward.

Several secondary results have also been presented throughout this paper. The most significant appears in the appendices and identifies the order to which the period may be determined when an asymptotically harmonic potential is approximated by a polynomial.

\section{Appendix A. Expansion of the lower turning point}

In this appendix we discuss the practical matter of how one can obtain approximate expressions for the period when the potential is such that $x_{-}$cannot be solved for explicitly in terms of $x_{+}$. Our method hinges on the fact that for Taylor expandable potentials, and for motion within a single well, the lower turning point's position will be given by a smooth function in $x_{+}$. The Taylor series for $x_{-} \equiv x_{-}\left(x_{+}\right)$, therefore, exists which implies that the lower turning point may be expanded in a power series in $x_{+}$. Assuming the origin is positioned at the potential's local minimum, it follows that $x_{-}$may be approximated as

$$
x_{-}=x_{+}\left(b_{0}+b_{1} x_{+}+\cdots+b_{n} x_{+}^{n}\right)+O\left(x_{+}^{n+2}\right) .
$$

Here, the coefficients $b_{i}$ are real and there is no constant term in the expansion since $x_{-}$must go to zero as $x_{+}$goes to zero. To determine these coefficients one must first plug (A.1) into the Taylor expansion of the equation $V\left(x_{-}\right)-V\left(x_{+}\right)=0$. Here, only terms up to order $2 m+n$ in $x_{+}$should be retained, where $2 m$ is the first positive integer for which $V^{(2 m)}(0) \neq 0$. This results in a polynomial of order $x_{+}^{2 m+n}$ for which all of the coefficients must be identically zero. In general, multiple sets of coefficients $b_{i}$ may be found that satisfy these coefficient equations. However, as the expansion is about a potential local minimum, it will always follow that $b_{0}=-1$, and the solution for the lower turning point will be uniquely determined.

Plugging the expansion for $x_{-}$into (3) and (5) allows one to obtain approximations for both $R(x)$ and $A\left(x_{+}\right)$. These approximations can then be plugged into (11) to obtain an expression for the period. A careful but straightforward look at the errors introduced through the above approximations can be made and indicates that for asymptotically harmonic potentials the resulting expression for the period will be valid at least to order $x_{+}^{n}$. By looking directly at the resulting expression for the period we show in appendix $B$ that this statement can be refined; given a Taylor series expansion for an asymptotically harmonic potential valid to order $x^{m}$ the above method may be applied to obtain an expression for the period valid to order $x_{+}^{2\lfloor m / 2\rfloor-1}$. In particular, this implies that the constant, harmonic approximation to the period is correct up to the first order. The linear term in the period expansion must always vanish for an asymptotically harmonic potential.

Use of expansion approximations for the lower turning point may often be fruitful for asymptotically anharmonic potentials as well. In this situation, however, it may not be clear 
from an analytic point of view how many terms in the series (11) should be retained and to what degree the approximations are valid.

For motions taking place within a multiple well potential, the lower turning point will be a piece-wise continuous function of $x_{+}$and will only take the form of (A.1) within the initial well. Outside this well a constant term must be added to the expansion of $x_{-}$and this may make the determination of the coefficients of the expansion difficult. By approximating the potential by a polynomial one may determine the coefficients of the lower turning point's expansion. This will allow one to obtain an expression for the period, but the expression will not be valid to any particular order in $x_{+}$.

\section{Appendix B. Even order potential approximations}

By applying the method of appendix A to the general fifth order potential approximation

$$
V(x)=x^{2}+v_{3} x^{3}+v_{4} x^{4}+v_{5} x^{5}+O\left(x^{6}\right),
$$

one obtains the following expression for the period:

$$
\frac{T}{\pi \sqrt{2 m}}=1+\frac{3}{16}\left(5 v_{3}^{2}-4 v_{4}\right) x_{+}^{2}+\frac{3}{16}\left(5 v_{3}^{3}-4 v_{3} v_{4}\right) x_{+}^{3}+O\left(x_{+}^{4}\right) .
$$

This result provides us with two interesting observations. The first is that the period contains no linear term in $x_{+}$. The second is that the result is indeed independent of $v_{5}$.

These observations may be generalized as follows. In the period expansion

$$
T=T_{0}+\cdots+T_{2 n-1} x_{+}^{2 n-1}+O\left(x_{+}^{2 n}\right),
$$

for the higher order generalization to the asymptotically harmonic potential (B.1), the term $T_{2 n-1}$ is independent of $v_{2 n+1}$. This implies that we may obtain an expression for the period valid to order $x_{+}^{2 n-1}$ if the potential is given up to order $x^{2 n}$.

To prove this result we first consider a potential whose expansion has been given up to order $2 n$. Assume for now that all terms in the expansion except for the quadratic and the term proportional to $x^{2 n}$ are zero, so that

$$
V(x)=x^{2}+v_{2 n} x^{2 n}+O\left(x^{2 n+1}\right) .
$$

For this potential we have

$$
\begin{aligned}
R(x) & =\sqrt{\frac{x_{+}^{2}-x^{2}}{x_{+}^{2}+v_{2 n} x_{+}^{2 n}-x^{2}-v_{2 n} x^{2 n}}} \\
& =\left[1+v_{2 n}\left(x^{2 n-2}+x^{2 n-4} x_{+}^{2}+\cdots+x_{+}^{2 n-2}\right)\right]^{-1 / 2} .
\end{aligned}
$$

In expression (11) for the period each coefficient $a_{i}$ in the expansion of $R(x)$ is multiplied by $A^{i}$. Since we are keeping terms only up to order $x_{+}^{2 n-2}$, we need only keep the first term in the binomial expansion of $R(x)$. Effectively,

$$
R(x)=1-\frac{v_{2 n}}{2}\left(x^{2 n-2}+x^{2 n-4} x_{+}^{2}+\cdots+x_{+}^{2 n-2}\right) .
$$

It follows that the period has the form

$$
T \propto 1+c v_{2 n} x_{+}^{2 n-2}+O\left(x_{+}^{2 n-1}\right),
$$

where $c$ is some nonzero, negative constant. The important fact that we observe here is that the coefficient $T_{2 n-2}$ is linear in $v_{2 n}$. For the more general asymptotically harmonic potential the period will have the form

$$
T=T_{0}+T_{1} x_{+}+\cdots+T_{2 n-2} x_{+}^{2 n-2}+O\left(x_{+}^{2 n-1}\right),
$$


where

$$
T_{2 n-2} \propto f_{2 n-2}\left(v_{3}, \ldots, v_{2 n-1}\right)+v_{2 n} .
$$

Here $f_{2 n-2}$ is independent of $v_{2 n}$ since terms involving the product of $v_{i}$ and $v_{2 n}$ will necessarily be of higher order in $x_{+}$. This is quite interesting because it implies that if

$$
v_{2 n}=-f_{2 n-2}\left(v_{3}, \ldots, v_{2 n-1}\right),
$$

the coefficient $T_{2 n-2}$ will be zero.

Now suppose we have found a potential for which the first nonconstant term in the expansion of the period is proportional to an odd power in $x_{+}$, so that

$$
T\left(x_{+}\right)=T_{0}+T_{2 n-1} x_{+}^{2 n-1}+O\left(x_{+}^{2 n}\right) .
$$

In analogy with the case of the even situation, this last coefficient will have the form

$$
T_{2 n-1}=f_{2 n-1}\left(v_{3}, \ldots, v_{2 n}\right)+\zeta v_{2 n+1},
$$

for some constant $\zeta$. Recall that $T\left(x_{+}\right)=T\left(x_{-}\right)$and that to lowest order we have $x_{-}=-x_{+}+O\left(x_{+}^{2}\right)$. Plugging these identities into (B.11) we find

$$
\begin{aligned}
T_{0}+T_{2 n-1} x_{+}^{2 n-1}+O\left(x_{+}^{2 n}\right) & =T_{0}+T_{2 n-1} x_{-}^{2 n-1}+O\left(x_{+}^{2 n}\right) \\
& =T_{0}-T_{2 n-1} x_{+}^{2 n-1}+O\left(x_{+}^{2 n}\right) .
\end{aligned}
$$

It follows that

$$
2 T_{2 n-1} x_{+}^{2 n-1}+O\left(x_{+}^{2 n}\right)=0
$$

so that

$$
T_{2 n-1}=f_{2 n-1}\left(v_{3}, \ldots, v_{2 n}\right)+\zeta v_{2 n+1}=0 .
$$

Since this identity always holds we conclude that $\zeta=0$ and that $T_{2 n-1}$ must be independent of $v_{2 n+1}$. By the above, we know that the even coefficients of the potential may be chosen so that the even coefficients in the period expansion are zero. An inductive step implies that we may thus choose the coefficients such that the period has the form (B.11) for any $n$. It therefore follows that $T_{2 n-1}$ is independent of $v_{2 n+1}$ for each integer $n$. This completes the proof.

As an application we present the following two potentials which were obtained using (B.2):

$$
V_{c}(x)=x^{2}+\alpha x^{3}+\frac{5 \alpha^{2}}{4} x^{4}+\beta x^{5}
$$

where $\alpha$ and $\beta$ are arbitrary constants, is a non-quadratic potential whose period is constant up to order $x_{+}^{4}$, and

$$
V_{1}(x)=1+x^{2}+\gamma x^{3}+\left(-\frac{4}{3}+\frac{5 \gamma^{2}}{4}\right) x^{4}+\cdots,
$$

with $\gamma$ an arbitrary constant, is a potential which has a period whose expansion in $x_{+}$is proportional to $V_{1}\left(x_{+}\right)$. It should be clear how to obtain higher-order potentials analogous to these two.

\section{References}

[1] Amore P and Fernandez F 2005 Exact and approximate expressions for the period of anharmonic oscillators Eur. J. Phys. 26 589-601

[2] Apostol T 1967 Calculus vol 1 (New York: Wiley)

[3] Chen T W 1980 Turning point approximation and application to nonlinear oscillators Am. J. Phys. 48 292-6

[4] Fernandez F M 1997 Perturbation theory in classical mechanics Eur. J. Phys. 18 436-9 
[5] Garfinkel B 1977 Theory of the Trojan asteroids: I Astron. J. 2 368-79

[6] Garfinkel B 1980 Theory of the Trojan asteroids: III Celest. Mech. 22 267-87

[7] Marion J B and Thornton S T 1995 Classical Dynamics of Particles and Systems (London: Harcourt Brace)

[8] Mohazzabi P 2004 Theory and examples of intrinsically nonlinear oscillators Am. J. Phys. 72 492-8

[9] Murray C D and Dermott S F 1999 Solar System Dynamics (Cambridge: Cambridge University Press)

[10] Rodriguez I and Brun J L 2000 A new method to compute the period of any anharmonic oscillator as a power series of the energy Eur. J. Phys. 21 617-23

[11] Zwillinger D 1996 CRC Standard Mathematical Tables and Formulae (Boca Raton, FL: CRC Press) 